\title{
Analysis of a Model for Computer Virus Transmission
}

\author{
Peng Qin ${ }^{1,2}$ \\ ${ }^{1}$ Institute of Signal Capturing and Processing Technology, North University of China, Taiyuan 030051, China \\ ${ }^{2}$ School of Computer Science and Control Engineering, North University of China, Taiyuan 030051, China
}

Correspondence should be addressed to Peng Qin; qinpeng@nuc.edu.cn

Received 28 May 2015; Revised 23 August 2015; Accepted 25 August 2015

Academic Editor: Ivanka Stamova

Copyright (C) 2015 Peng Qin. This is an open access article distributed under the Creative Commons Attribution License, which permits unrestricted use, distribution, and reproduction in any medium, provided the original work is properly cited.

\begin{abstract}
Computer viruses remain a significant threat to computer networks. In this paper, the incorporation of new computers to the network and the removing of old computers from the network are considered. Meanwhile, the computers are equipped with antivirus software on the computer network. The computer virus model is established. Through the analysis of the model, diseasefree and endemic equilibrium points are calculated. The stability conditions of the equilibria are derived. To illustrate our theoretical analysis, some numerical simulations are also included. The results provide a theoretical basis to control the spread of computer virus.
\end{abstract}

\section{Introduction}

With the rapid development of computer, communication, and network technology, network information system has become an important way of the development of countries and industries, amongst others. Information security has become one of the most important and challenging issues faced in the age of information sharing. The computer virus is one common information security threat.

Computer virus is not only destructive, but also highly contagious. Once the virus is copied or generates variety, its speed is difficult to be controlled. Infectivity is the basic characteristic of the virus. In biology, the virus can diffuse from one organism to another. Cohen, Kephart and White pointed out that the spread mechanisms of computer viruses and biological viruses have many similarities $[1,2]$. Under appropriate conditions, biological viruses can multiply quickly, and the infected organisms exhibit symptoms or even die. Similarly, the computer virus can also spread to uninfected computers from the infected computer through many kinds of ways. In some cases, the infected computers do not work and even get paralysed. Unlike biological viruses, the computer virus is a software program designed to replicate itself and spread to other machines. Computer virus enters the computer and gets executed; it will search for other programs or storage media in line with the conditions of their infection and target and then insert the code itself, achieving the purpose of self-reproduction. As long as a computer is infected (if not promptly treated), the virus will spread rapidly on this computer, in which a large number of files (usually an executable file) will be infected. The infected file has become a new source of infection and then will exchange data with other machines or over the network exposure; the virus will continue to be contagious. A computer virus can enter your computer in any number of ways, such as via mobile hard disk, via an email attachment, during file downloads from the Internet, or even upon a visit to a contaminated web site. By the time you find the virus that infected the computer, the mobile hard disk which is often used on this computer has been infected with the virus. Other computers connected to the machine network might be infected with the virus. The network has no permanent immunity to the computer virus. Therefore, there is always computer virus.

Kephart et al. [2,3] study the spread of computer virus using the biological virus model; they mainly focus on the influence of network topology on the spread of computer virus. In the homogeneous mixing nodes and only susceptible node input cases, Mishra et al. $[4,5]$ establish the mathematical model of Internet spread of computer virus. They analyze the propagation law of virus using threshold theory of infectious disease dynamics and predict the development trend of computer virus. Piqueira et al. [6] study the effects 
of equipping antivirus software on the virus in computer network. They prove the stability of the disease-free equilibrium and endemic equilibrium without incorporating new computers and removing old computers. For more studies on virus in computer network, see [7-13] and so on.

In this paper, the new computers are incorporated to the network, and the old computers are removed from the computer network. Meanwhile, the computers are equipped with antivirus software on the computer network. The remainder of this paper presents the model and results. In Section 2, the mathematical model on computer virus is described, and in Section 3 we obtain the equilibriums. In Section 4, the analysis of this model is derived. Numerical simulations supporting the theoretical analysis are given in Section 5 . The paper ends with a conclusion and discussion in Section 6.

\section{The Dynamic Model}

The model proposed here is based on the compartmental SAIR model [6-8], including an antidotal population compartment $(A)$ representing nodes of the network equipped with fully effective antivirus programs, a susceptible compartment $(S)$, an infective compartment $(I)$, and a temporarily immune compartment $(R)$. Connections between the compartments represent operational parameters of the network and their control can be used as a strategy to maintain the reliability of the whole system, even in the presence of infections (see Figure 1).

The total population $N$ is divided into four groups. $S(t)$ denote noninfected computers subjected to possible infection. $I(t)$ denote infected computers. $R(t)$ denote removed computers due to infection or not. $A(t)$ denote noninfected computers equipped with antivirus.

The SAIR model for computer viruses propagation was proposed and can be described by

$$
\begin{aligned}
& \frac{d S(t)}{d t}=C-\alpha_{S A} S A-\beta S I-\mu S+\sigma R, \\
& \frac{d I(t)}{d t}=\beta S I-\alpha_{I A} I A-\delta I-\mu I, \\
& \frac{d R(t)}{d t}=\delta I-\sigma R-\mu R, \\
& \frac{d A(t)}{d t}=\alpha_{S A} S A+\alpha_{I A} I A-\mu A,
\end{aligned}
$$

where $C$ is influx rate, representing the incorporation of new computers to the network; $\beta$ is infection rate of susceptible computers; $\mu$ is proportion coefficient for the mortality rate, not due to the virus; $\delta$ is removal rate of infected computers; $\sigma$ is recovering rate of removed computers, with an operator intervention; $\alpha_{S A}$ is conversion of susceptible computers into antidotal ones, occurring when susceptible computers establish effective communication with antidotal ones and the antidotal one installs the antivirus in the susceptible ones; $\alpha_{I A}$ represents infected computers that can be fixed by using antivirus programs being converted into antidotal ones.

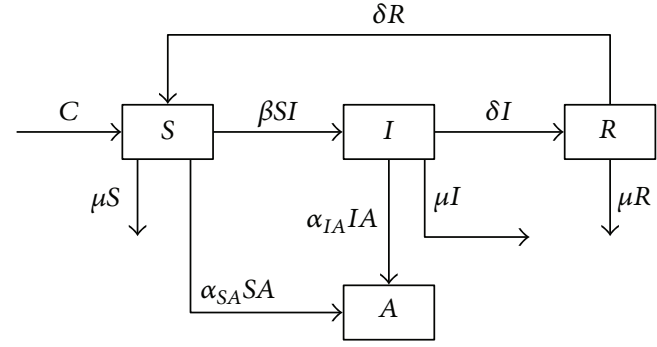

Figure 1: Transfer diagram of the model.

Let $N=S+I+R+A$; then $d N(t) / d t=C-\mu N$. Let

$\Omega$

$$
=\left\{(S, I, R, A): S, I, R, A \leq 0, S+I+R+A \leq \frac{C}{\mu}\right\} .
$$

Then, it is clear that $\Omega$ is a positive invariant set. Hence, we will focus our attention only on the region $\Omega$.

But $[6,7]$ think the influx rate is considered to be $C=0$, $\mu=0$. In fact, every day new computers can be incorporated to the network or old computers can be removed from the network. Therefore, they can not be ignored. In this paper, we analyse completely dynamical behavior of the spread of the virus in computer network with incorporating new computers and removing old computers.

\section{The Disease-Free and Endemic Equilibrium}

When $I=0$, if $A=0$, the disease-free equilibrium of system (1) is $P_{1}=\left(S_{1}, I_{1}, R_{1}, A_{1}\right)=(C / \mu, 0,0,0)$. If $A \neq 0$, we obtain the threshold $R_{01}=C \alpha_{S A} / \mu^{2}$. When $R_{01}>1$, the diseasefree equilibrium of system (1) is $P_{2}=\left(S_{2}, I_{2}, R_{2}, A_{2}\right)=$ $\left(\mu / \alpha_{S A}, 0,0, C / \mu-\mu / \alpha_{S A}\right)$.

In the following, we compute the positive equilibrium; namely, $I \neq 0$.

According to the third equation of system (1), we have

$$
R=\frac{\delta}{\sigma+\mu} I
$$

When $A=0$, using the second equation of system (1), we have

$$
S=\frac{\delta+\mu}{\beta}
$$

According to the first equation of system (1), we obtain

$$
I=\frac{(\beta C-\mu(\delta+\mu))(\sigma+\mu)}{\beta \mu(\sigma+\delta+\mu)} .
$$


Therefore, when the threshold $R_{02}=\beta C / \mu(\delta+\mu)>1$, we have the positive equilibrium

$$
\begin{aligned}
P_{3} & =\left(S_{3}, I_{3}, R_{3}, A_{3}\right) \\
& =\left(\frac{\delta+\mu}{\beta}, \frac{\left(R_{02}-1\right)(\delta+\mu)(\sigma+\mu)}{\beta(\sigma+\delta+\mu)}, \frac{\delta}{\sigma+\mu} I_{3}, 0\right) .
\end{aligned}
$$

When $A \neq 0$, taking advantage of the second equation of system (1), we have

$$
A=\frac{\beta S-(\delta+\mu)}{\alpha_{I A}}
$$

According to the fourth equation of system (1), we have

$$
I=\frac{\mu-\alpha_{S A} S}{\alpha_{I A}} .
$$

Substituting (7) and (8) into the first equation of system (1), we have

$$
\begin{gathered}
C-\alpha_{S A} S \frac{\beta S-(\delta+\mu)}{\alpha_{I A}}-\beta S \frac{\mu-\alpha_{S A} S}{\alpha_{I A}}-\mu S \\
+\frac{\sigma \delta}{\sigma+\mu} \frac{\mu-\alpha_{S A} S}{\alpha_{I A}}=0 .
\end{gathered}
$$

Namely,

$$
\begin{gathered}
{\left[\beta \mu+\alpha_{I A} \mu+\frac{\sigma \delta \alpha_{S A}}{\sigma+\mu}-(\delta+\mu) \alpha_{S A}\right] S} \\
=C \alpha_{I A}+\frac{\sigma \delta \mu}{\sigma+\mu} .
\end{gathered}
$$

Hence,

$$
S=\frac{C \alpha_{I A}+\sigma \delta \mu /(\sigma+\mu)}{\beta \mu+\alpha_{I A} \mu+\sigma \delta \alpha_{S A} /(\sigma+\mu)-(\delta+\mu) \alpha_{S A}} .
$$

In order to make $S>0$, it must satisfy the following conditions:

$$
\beta \mu+\alpha_{I A} \mu+\frac{\sigma \delta \alpha_{S A}}{\sigma+\mu}-(\delta+\mu) \alpha_{S A}>0
$$

Namely,

$$
R_{03}=\frac{\beta \mu+\alpha_{I A} \mu+\sigma \delta \alpha_{S A} /(\sigma+\mu)}{(\delta+\mu) \alpha_{S A}}>1 .
$$

Therefore, if the threshold $R_{03}>1$, we obtain

$$
\begin{aligned}
S_{4} & =\frac{C \alpha_{I A}+\sigma \delta \mu /(\sigma+\mu)}{(\delta+\mu) \alpha_{S A}\left(R_{03}-1\right)}, \\
I_{4} & =\frac{\mu-\alpha_{S A} S_{4}}{\alpha_{I A}}, \\
A_{4} & =\frac{\beta S_{4}-\delta-\mu}{\alpha_{I A}} .
\end{aligned}
$$

In order to make $I_{4}, A_{4}>0$, it must satisfy

$$
\begin{aligned}
& R_{03}>1+\frac{C \alpha_{I A}(\sigma+\mu)+\sigma \delta \mu}{\mu(\sigma+\mu)(\delta+\mu)}, \\
& R_{03}<1+\frac{\beta \mu}{(\delta+\mu) \alpha_{S A}} \frac{C \alpha_{I A}(\sigma+\mu)+\sigma \delta \mu}{\mu(\sigma+\mu)(\delta+\mu)} .
\end{aligned}
$$

When $1+\left(C \alpha_{I A}(\sigma+\mu)+\sigma \delta \mu\right) / \mu(\sigma+\mu)(\delta+\mu)<R_{03}<$ $1+\left(\beta \mu /(\delta+\mu) \alpha_{S A}\right)\left(\left(C \alpha_{I A}(\sigma+\mu)+\sigma \delta \mu\right) / \mu(\sigma+\mu)(\delta+\mu)\right)$ (namely, the threshold $R_{04}=\beta \mu /(\delta+\mu) \alpha_{S A}>1$ ), we have the positive equilibrium

$$
\begin{aligned}
P_{4} & =\left(S_{4}, I_{4}, R_{4}, A_{4}\right) \\
& =\left(\frac{C \alpha_{I A}+\sigma \delta \mu /(\sigma+\mu)}{(\delta+\mu) \alpha_{S A}\left(R_{03}-1\right)}, \frac{\mu-\alpha_{S A} S_{4}}{\alpha_{I A}}, \frac{\delta}{\sigma+\mu}\right. \\
& \left.\cdot I_{4}, \frac{\beta S_{4}-\delta-\mu}{\alpha_{I A}}\right) .
\end{aligned}
$$

\section{Stability of Equilibrium}

4.1. Stability of the Disease-Free Equilibrium. The Jacobin matrix of system (1) at the disease-free equilibrium $P_{1}$ is

$$
\begin{aligned}
& J\left(P_{1}\right) \\
& =\left(\begin{array}{cccc}
-\mu & -\frac{\beta C}{\mu} & \sigma & -\frac{C \alpha_{S A}}{\mu} \\
0 & \frac{\beta C}{\mu}-\delta-\mu & 0 & 0 \\
0 & \delta & -(\sigma+\mu) & 0 \\
0 & 0 & 0 & \frac{C \alpha_{S A}}{\mu}-\mu
\end{array}\right) .
\end{aligned}
$$

The characteristic equation of $J\left(P_{1}\right)$ is given by

$$
(\lambda+\mu)\left(\lambda+l_{1}\right)(\lambda+\sigma+\mu)\left(\lambda+l_{2}\right)=0,
$$

where $l_{1}=-\beta C / \mu+\delta+\mu$ and $l_{2}=-C \alpha_{S A} / \mu+\mu$. Therefore, we have $l_{1}>0$ if and only if $R_{02}=\beta C / \mu(\delta+\mu)<1$, and $l_{2}>0$ if and only if $R_{01}=C \alpha_{S A} / \mu^{2}<1$. It follows from the RouthHurwitz criterion that the eigenvalues have negative real parts if and only if $R_{01}<1$ and $R_{02}<1$. Hence, the disease-free equilibrium $P_{1}$ of model (1) is locally asymptotically stable if $R_{01}<1$ and $R_{02}<1$ and unstable if $R_{01}>1$ or $R_{02}>1$.

If $R_{01}>1$, the disease-free equilibrium $P_{1}$ is unstable, and system (1) exhibits the other disease-free equilibrium $P_{2}$. 
If $R_{02}>1$, the disease-free equilibrium $P_{2}$ is unstable, and system (1) exhibits the positive equilibrium $P_{3}$.
The Jacobin matrix of system (1) at the disease-free equilibrium $P_{2}$ is

$$
J\left(P_{2}\right)=\left(\begin{array}{cccc}
-\mu-\alpha_{S A} A_{2} & -\beta S_{2} & \sigma & -\alpha_{S A} S_{2} \\
0 & \beta S_{2}-\alpha_{I A} A_{2}-\delta-\mu & 0 & 0 \\
0 & \delta & -(\sigma+\mu) & 0 \\
\alpha_{S A} A_{2} & \alpha_{I A} A_{2} & 0 & \alpha_{S A} S_{2}-\mu
\end{array}\right) .
$$

Since $\alpha_{S A} S_{2}-\mu=0$, the characteristic equation of $J\left(P_{2}\right)$ is given by

$$
\begin{gathered}
{[\lambda+(\sigma+\mu)]\left[\lambda-\left(\beta S_{2}-\alpha_{I A} A_{2}-\delta-\mu\right)\right]} \\
\cdot\left[\lambda^{2}+\left(\mu+\alpha_{S A} S_{2}\right) \lambda+\mu \alpha_{S A} S_{2}\right]=0 .
\end{gathered}
$$

By calculating, we obtain that the characteristic equation has the four eigenvalues: $-(\sigma+\mu), \beta S_{2}-\alpha_{I A} A_{2}-\delta-$ $\mu,-\mu$, and $-\alpha_{S A} A_{2}$. When $R_{01}>1,-\alpha_{S A} A_{2}<0$. We have $\beta S_{2}-\alpha_{I A} A_{2}-\delta-\mu<0$ if and only if $R_{05}=(\beta+$ $\left.\alpha_{I A}\right) \mu^{2} /\left(C \alpha_{S A} \alpha_{I A}+\alpha_{S A}(\delta+\mu)\right)<1$. Hence, the disease-free equilibrium $P_{2}$ of model (1) is locally asymptotically stable if $R_{01}>1$ and $R_{05}<1$. The disease-free equilibrium $P_{2}$ of model (1) is unstable if $R_{01}>1$ and $R_{05}>1$.

Theorem 1. (1) If $R_{01}<1$ and $R_{02}<1$, the disease-free equilibrium $P_{1}$ of system (1) is locally asymptotically stable. If $R_{01}>1$ or $R_{02}>1$, the disease-free equilibrium $P_{1}$ of system (1) is unstable.

(2) If $R_{01}>1$, the disease-free equilibrium $P_{1}$ is unstable, and system (1) exhibits the other disease-free equilibrium $P_{2}$.

(3) If $R_{02}>1$, the disease-free equilibrium $P_{1}$ is unstable, and system (1) exhibits the positive equilibrium $P_{3}$.

(4) If $R_{01}>1$ and $R_{05}<1$, the disease-free equilibrium $P_{2}$ is locally asymptotically stable. If $R_{05}>1$, the disease-free equilibrium $P_{2}$ is unstable.

4.2. Stability of the Endemic Equilibrium. In the following, we study the stability of the endemic equilibriums.

The Jacobin matrix of system (1) at the endemic equilibrium $P_{3}$ is

$$
\begin{aligned}
& J\left(P_{3}\right) \\
& =\left(\begin{array}{cccc}
-\beta I_{3}-\mu & -\beta S_{3} & \sigma & -\alpha_{S A} S_{3} \\
\beta I_{3} & \beta S_{3}-\delta-\mu & 0 & -\alpha_{I A} I_{3} \\
0 & \delta & -(\sigma+\mu) & 0 \\
0 & 0 & 0 & \alpha_{S A} S_{3}+\alpha_{I A} I_{3}-\mu
\end{array}\right) .
\end{aligned}
$$

Since $\beta S_{3}-\delta-\mu=0$, the characteristic equation of $J\left(P_{3}\right)$ is given by

$$
\left(\lambda-\left(\alpha_{S A} S_{3}+\alpha_{I A} I_{3}-\mu\right)\right) F(\lambda)=0,
$$

where

$$
\begin{aligned}
F(\lambda) & =\lambda^{3}+a_{1} \lambda^{2}+a_{2} \lambda+a_{3}, \\
a_{1} & =\beta I_{3}+\mu+\sigma+\mu>0, \\
a_{2} & =\left(\beta I_{3}+\mu\right)(\sigma+\mu)+\beta^{2} S_{3} I_{3}>0, \\
a_{3} & =\beta^{2} S_{3} I_{3}(\sigma+\mu)-\beta \sigma \delta I_{3} \\
& =\beta I_{3}[(\delta+\mu)(\sigma+\mu)-\delta \sigma]>0 .
\end{aligned}
$$

It is easy to calculate $a_{1} a_{2}-a_{3}>0$. Hence, the eigenvalues of $F(\lambda)=0$ have negative real parts.

Therefore, if the eigenvalues of the characteristic equation in $P_{3}$ want to have negative real parts, only $\alpha_{S A} S_{3}+\alpha_{I A} I_{3}-\mu<$ 0 . Namely,

$$
\begin{aligned}
& R_{02} \\
& <1 \\
& +\frac{\alpha_{S A}(\sigma+\delta+\mu)(\delta+\mu)\left(\beta \mu / \alpha_{S A}(\delta+\mu)-1\right)}{\alpha_{I A}(\delta+\mu)(\sigma+\mu)} \\
& =R_{07} \text {. }
\end{aligned}
$$

According to Routh-Hurwitz criterion, the epidemic equilibrium $P_{3}$ is locally asymptotically stable if $1<R_{02}<R_{07}$ and unstable if $R_{02}>R_{07}$.

System (1) has the following limiting system:

$$
\begin{aligned}
& \frac{d I(t)}{d t}=\beta\left(\frac{C}{\mu}-I-R-A\right) I-\alpha_{I A} A I-\delta I-\mu I, \\
& \frac{d R(t)}{d t}=\delta I-\sigma R-\mu R, \\
& \frac{d A(t)}{d t}=\alpha_{S A}\left(\frac{C}{\mu}-I-R-A\right) A+\alpha_{I A} A I-\mu A .
\end{aligned}
$$

In the following, we prove the stability of the endemic equilibrium $P_{4}$ by the limit system. The Jacobin matrix of system (25) at the endemic equilibrium $P_{4}$ is 


$$
J\left(P_{4}\right)=\left(\begin{array}{ccc}
\beta S_{4}-\beta I_{4}-\alpha_{I A} A_{4}-\mu-\delta & -\beta I_{4} & -\beta I_{4}-\alpha_{I A} I_{4} \\
\delta & -(\sigma+\mu) & 0 \\
-\alpha_{S A} A_{4}+\alpha_{I A} A_{4} & -\alpha_{S A} A_{4} & \alpha_{S A} S_{4}-\alpha_{S A} A_{4}+\alpha_{I A} I_{4}-\mu
\end{array}\right)
$$

Because $\beta S_{4}-\alpha_{I A} A_{4}-\mu-\delta=0, \alpha_{S A} S_{4}+\alpha_{I A} I_{4}-\mu=0$, the above matrix becomes

$$
\begin{aligned}
& J\left(P_{4}\right) \\
& =\left(\begin{array}{ccc}
-\beta I_{4} & -\beta I_{4} & -\beta I_{4}-\alpha_{I A} I_{4} \\
\delta & -(\sigma+\mu) & 0 \\
-\alpha_{S A} A_{4}+\alpha_{I A} A_{4} & -\alpha_{S A} A_{4} & -\alpha_{S A} A_{4}
\end{array}\right) .
\end{aligned}
$$

The characteristic equation of $J\left(P_{4}\right)$ is given by

$$
\lambda^{3}+b_{1} \lambda^{2}+b_{2} \lambda+b_{3}=0
$$

where

$$
\begin{aligned}
b_{1}= & \beta I_{4}+\sigma+\mu+\alpha_{S A} A_{4}>0, \\
b_{2}= & \alpha_{S A} \beta I_{4} A_{4}+\alpha_{S A} A_{4}(\sigma+\mu)+\beta I_{4}(\sigma+\mu) \\
& -\left(\alpha_{S A} A_{4}-\alpha_{I A} A_{4}\right)\left(\beta I_{4}+\alpha_{I A} I_{4}\right)+\delta \beta I_{4}, \\
b_{3}= & \alpha_{S A} \beta I_{4} A_{4}(\sigma+\mu)-\delta \alpha_{S A} A_{4}\left(\beta I_{4}+\alpha_{I A} I_{4}\right) \\
& -(\sigma+\mu)\left(\alpha_{S A} A_{4}-\alpha_{I A} A_{4}\right)\left(\beta I_{4}+\alpha_{I A} I_{4}\right) \\
& +\delta \alpha_{S A} \beta I_{4} A_{4} .
\end{aligned}
$$

Because of $\alpha_{I A} I_{4}=\mu-\alpha_{S A} S_{4}$, we have

$$
\begin{aligned}
b_{2}= & \alpha_{S A} \beta I_{4} A_{4}+\alpha_{S A} A_{4}(\sigma+\mu)+\beta I_{4}(\sigma+\mu) \\
& -\left(\alpha_{S A} A_{4}-\alpha_{I A} A_{4}\right)\left(\beta I_{4}+\alpha_{I A} I_{4}\right)+\delta \beta I_{4} \\
= & \alpha_{S A} A_{4}(\sigma+\mu)+\beta I_{4}(\sigma+\mu)-\alpha_{S A} A_{4} \alpha_{I A} I_{4} \\
& +\alpha_{I A} A_{4}\left(\beta I_{4}+\alpha_{I A} I_{4}\right)+\delta \beta I_{4} \\
= & \alpha_{S A} A_{4}(\sigma+\mu)+\beta I_{4}(\sigma+\mu) \\
& -\alpha_{S A} A_{4}\left(\mu-\alpha_{S A} S_{4}\right)+\alpha_{I A} A_{4}\left(\beta I_{4}+\alpha_{I A} I_{4}\right) \\
& +\delta \beta I_{4} \\
= & \alpha_{S A} A_{4} \sigma+\beta I_{4}(\sigma+\mu)+\alpha_{S A} A_{4} \alpha_{S A} S_{4} \\
& +\alpha_{I A} A_{4}\left(\beta I_{4}+\alpha_{I A} I_{4}\right)+\delta \beta I_{4}>0 . \\
b_{3}= & \alpha_{S A} \beta I_{4} A_{4}(\sigma+\mu)-\delta \alpha_{S A} A_{4}\left(\beta I_{4}+\alpha_{I A} I_{4}\right) \\
& -(\sigma+\mu)\left(\alpha_{S A} A_{4}-\alpha_{I A} A_{4}\right)\left(\beta I_{4}+\alpha_{I A} I_{4}\right) \\
& +\delta \alpha_{S A} \beta I_{4} A_{4} \\
= & \alpha_{I A} A_{4} I_{4}(\sigma+\mu)\left(\beta+\alpha_{I A}\right)-\delta \alpha_{S A} A_{4} \alpha_{I A} I_{4} \\
& -(\sigma+\mu) \alpha_{S A} \alpha_{I A} A_{4} I_{4} . \\
& \left(\sigma{ }_{1} .\right.
\end{aligned}
$$

It is easy to calculate $b_{1} b_{2}-b_{3}>0$. Therefore, in order to make $b_{3}>0$, only there is $R_{06}=(\sigma+\mu)\left(\beta+\alpha_{I A}\right) / \alpha_{S A}(\sigma+\delta+\mu)>1$. Hence, if the threshold $R_{06}=(\sigma+\mu)\left(\beta+\alpha_{I A}\right) / \alpha_{S A}(\sigma+\delta+\mu)>$ 1 , the endemic equilibrium $P_{4}$ is locally asymptotically stable.

Theorem 2. (1) If $1<R_{02}<R_{07}$, the positive equilibrium $P_{3}$ is locally asymptotically stable. If $R_{02}>R_{07}$, the positive equilibrium $P_{3}$ is unstable.

(2) If $1+\left(C \alpha_{I A}(\sigma+\mu)+\sigma \delta \mu\right) / \mu(\sigma+\mu)(\delta+\mu)<R_{03}<$ $1+\left(\beta \mu /(\delta+\mu) \alpha_{S A}\right)\left(\left(C \alpha_{I A}(\sigma+\mu)+\sigma \delta \mu\right) / \mu(\sigma+\mu)(\delta+\mu)\right)$ and $R_{06}=(\sigma+\mu)\left(\beta+\alpha_{I A}\right) / \alpha_{S A}(\sigma+\delta+\mu)>1$, the positive equilibrium $P_{4}$ is locally asymptotically stable. If $R_{06}=(\sigma+$ $\mu)\left(\beta+\alpha_{I A}\right) / \alpha_{S A}(\sigma+\delta+\mu)<1$, the positive equilibrium $P_{4}$ is unstable.

\section{Numerical Simulation}

In this section, we will perform a series of numerical simulations to verify the mathematical analysis. In Figure 2, when $R_{01}<1$ and $R_{02}<1$, the disease-free equilibrium $P_{1}$ is asymptotically stable, where the initial values are $S=50$, $I=20, R=1$, and $A=1$. The parameters are $C=1$, $\alpha_{S A}=0.00045, \beta=0.05, \mu=0.05, \sigma=0.8, \alpha_{I A}=0.0025$, and $\delta=0.96$.

If $R_{01}>1$ and $R_{05}<1$, Figure 3 signifies that the diseasefree equilibrium $P_{2}$ of system (1) is asymptotically stable. The initial values are $S=50, I=20, R=1$, and $A=1$. Choose the parameters as follows: $C=6, \alpha_{S A}=0.0045, \beta=0.1, \mu=0.05$, $\sigma=0.8, \alpha_{I A}=0.0025$, and $\delta=0.8$.

If $1<R_{02}<R_{07}$, the positive equilibrium $P_{3}$ of system (1) is asymptotically stable (see Figure 4 ). The initial values are $S=50, I=20, R=1$, and $A=1$. Choose the parameters as follows: $C=1, \alpha_{S A}=0.00045, \beta=0.2, \mu=0.05, \sigma=0.8$, $\alpha_{I A}=0.0025$, and $\delta=0.96$.

If $1+\left(C \alpha_{I A}(\sigma+\mu)+\sigma \delta \mu\right) / \mu(\sigma+\mu)(\delta+\mu)<R_{03}<1+$ $\left(\beta \mu /(\delta+\mu) \alpha_{S A}\right)\left(\left(C \alpha_{I A}(\sigma+\mu)+\sigma \delta \mu\right) / \mu(\sigma+\mu)(\delta+\mu)\right)$ and $R_{06}=$ $(\sigma+\mu)\left(\beta+\alpha_{I A}\right) / \alpha_{S A}(\sigma+\delta+\mu)>1$, the positive equilibrium $P_{4}$ of system (1) is asymptotically stable (see Figure 5 ). The initial values are $S=50, I=20, R=1$, and $A=1$. Choose the parameters as follows: $C=10, \alpha_{S A}=0.00045, \beta=0.2$, $\mu=0.05, \sigma=0.8, \alpha_{I A}=0.0025$, and $\delta=17$.

\section{Conclusion}

This paper mainly considers the incorporation of new computers to the network, the removal of old computers from the network, the computer equipped with antivirus software, and so forth. They affect the spread of the virus. The model for computer virus transmission is established. Through the analysis of the model, two disease-free and two positive equilibriums are obtained. The stability conditions of the equilibriums are derived. 


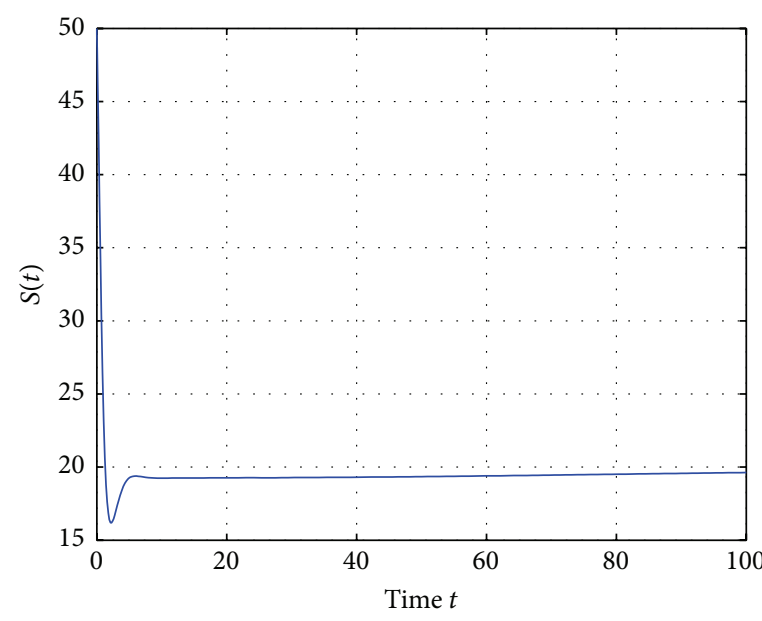

$-S(t)$

(a)

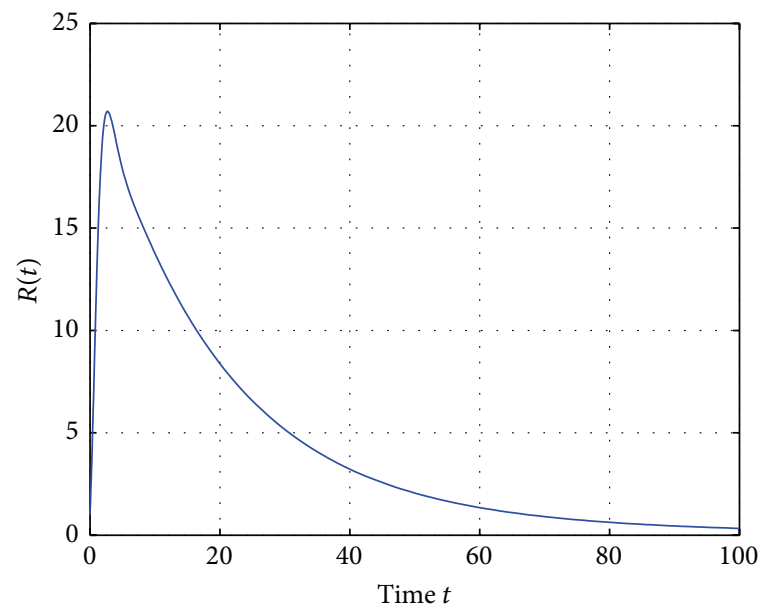

$-R(t)$

(c)
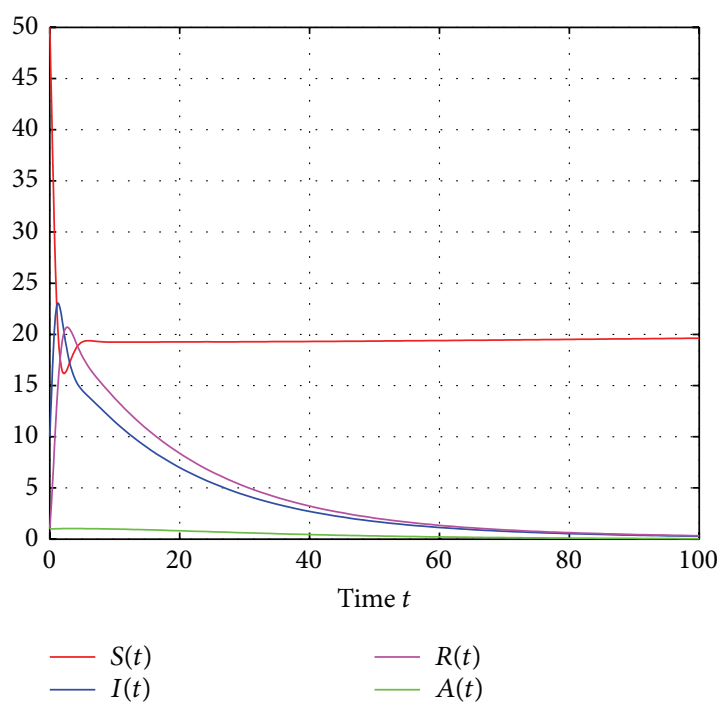

(e)

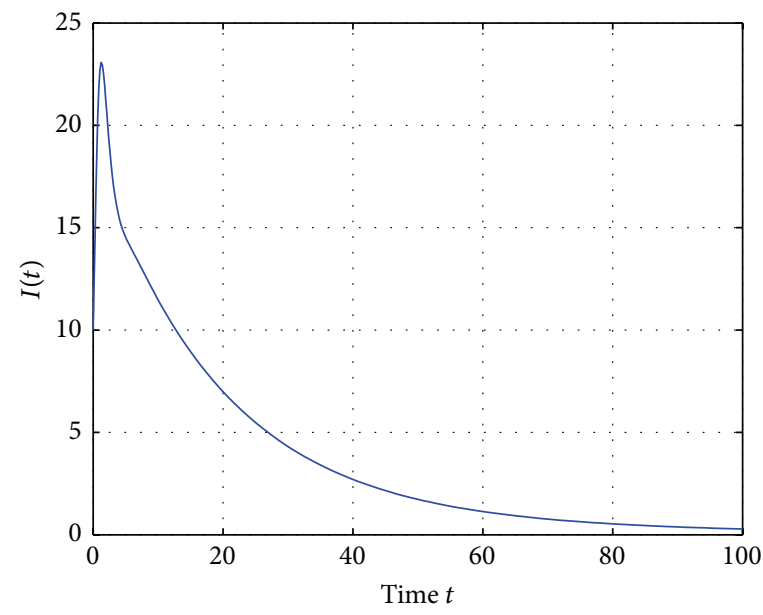

$-I(t)$

(b)

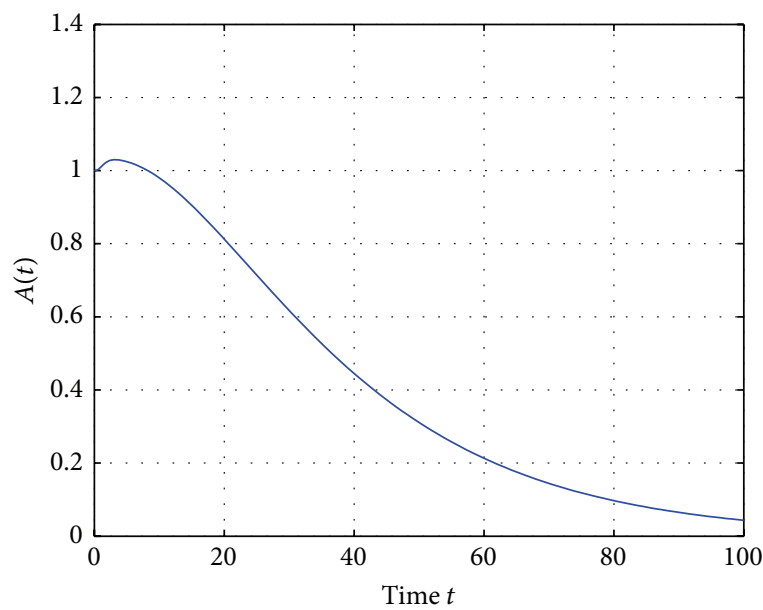

$-A(t)$

(d)

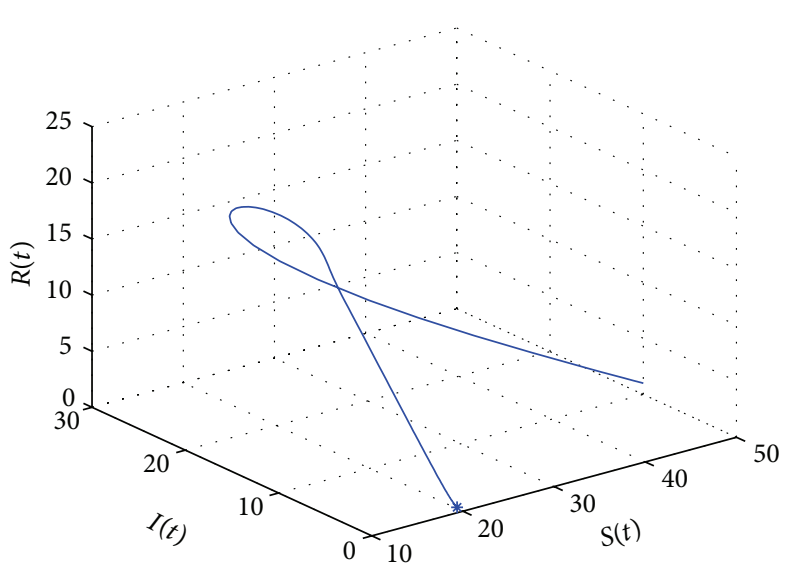

(f)

FIGURE 2: $R_{01}<1$ and $R_{02}<1$. The disease-free equilibrium $P_{1}$ of system (1) is asymptotically stable. 


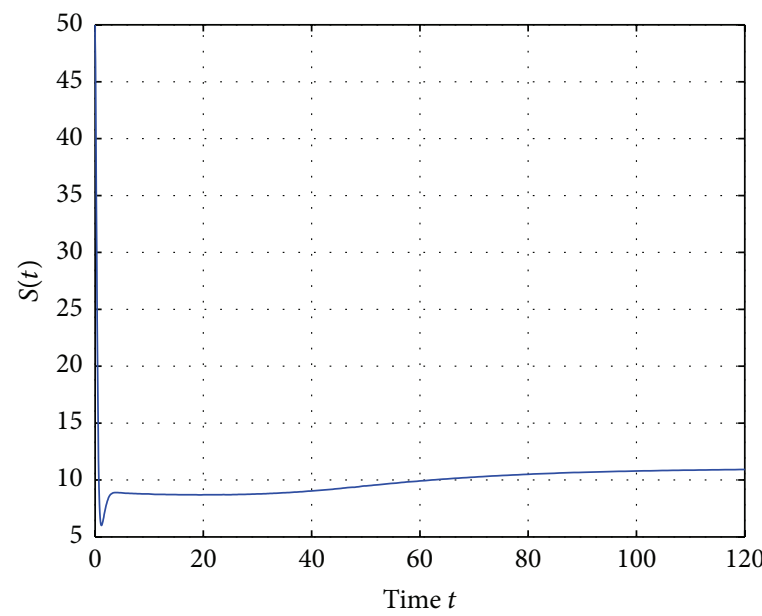

$-S(t)$

(a)

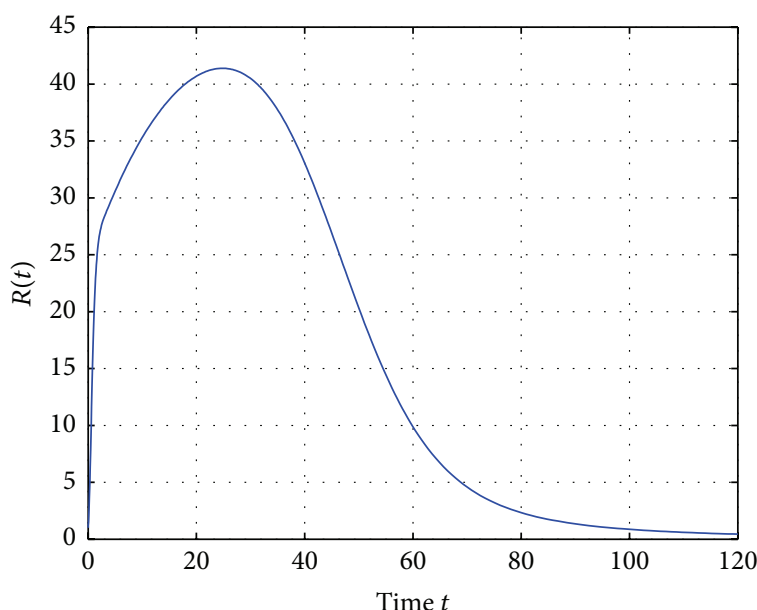

$-R(t)$

(c)

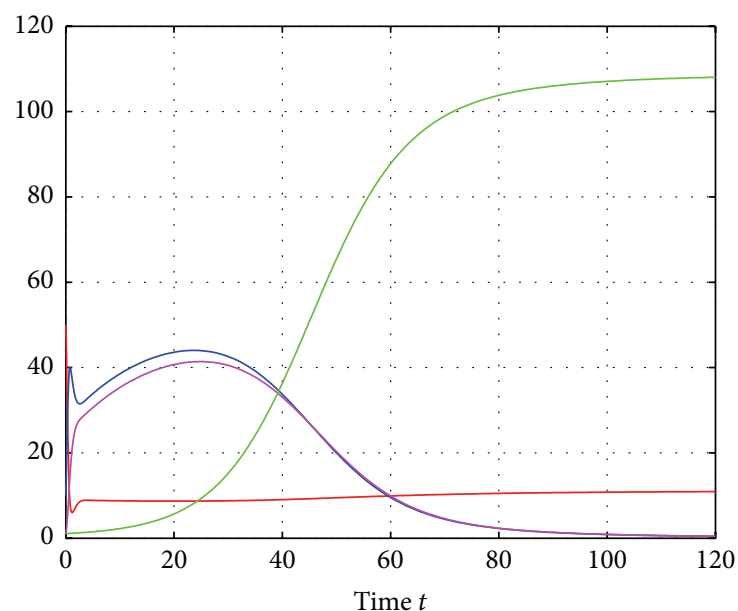

$\begin{array}{ll}-S(t) & -R(t) \\ -I(t) & -A(t)\end{array}$

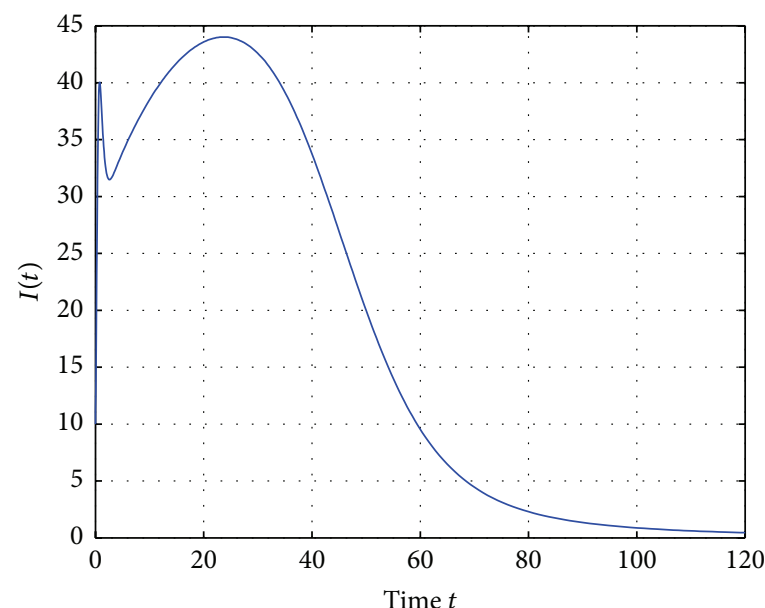

$-I(t)$

(b)

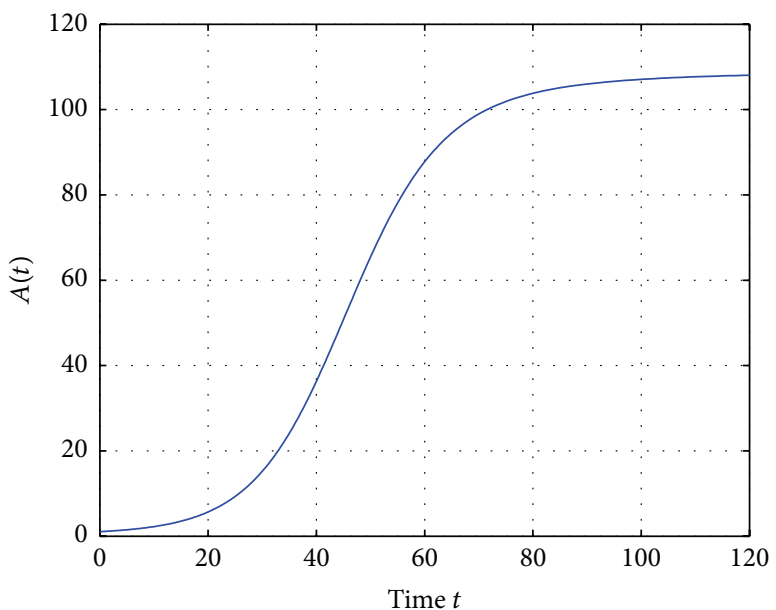

$-A(t)$

(d)

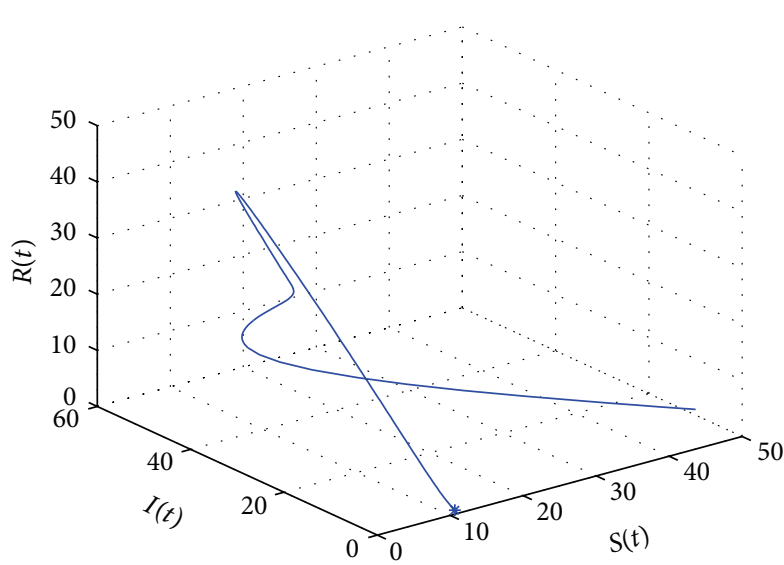

(e)

(f)

FIgURE 3: $R_{01}>1$ and $R_{05}<1$. The disease-free equilibrium $P_{2}$ of system (1) is asymptotically stable. 

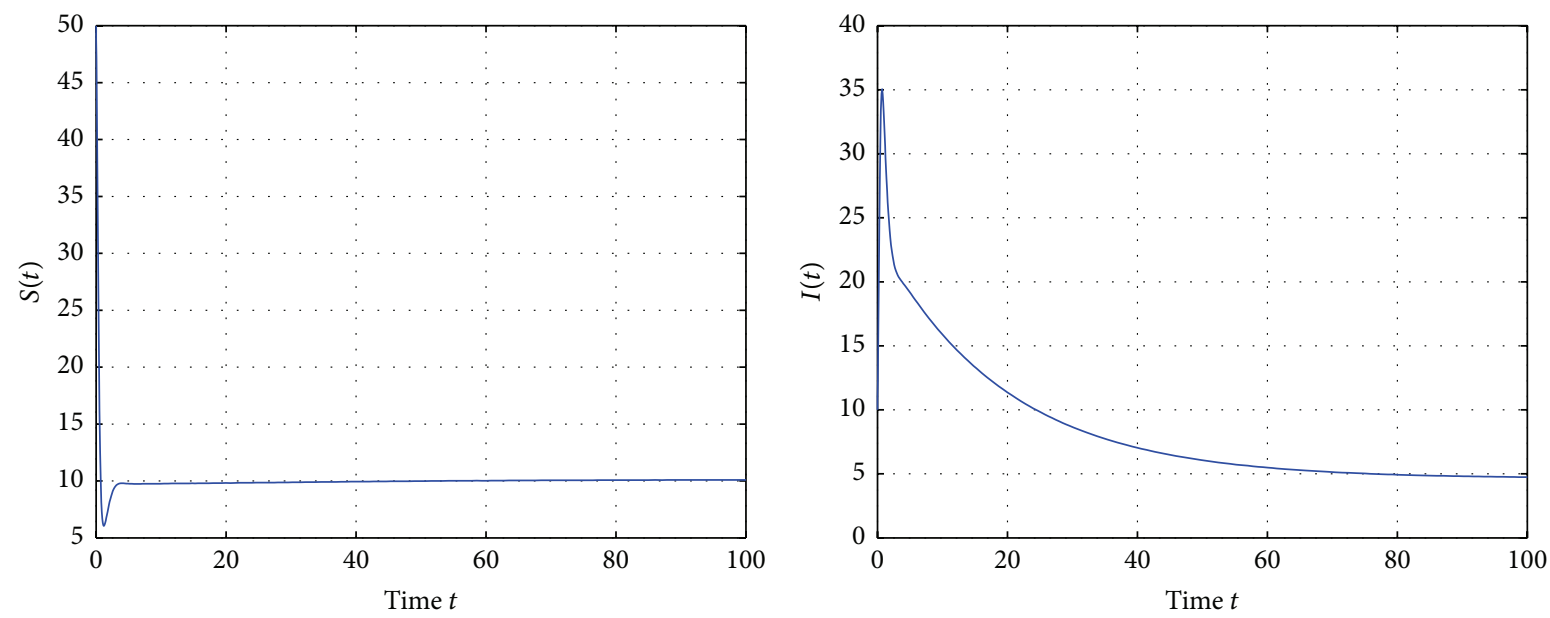

$-S(t)$ $-I(t)$

(a)
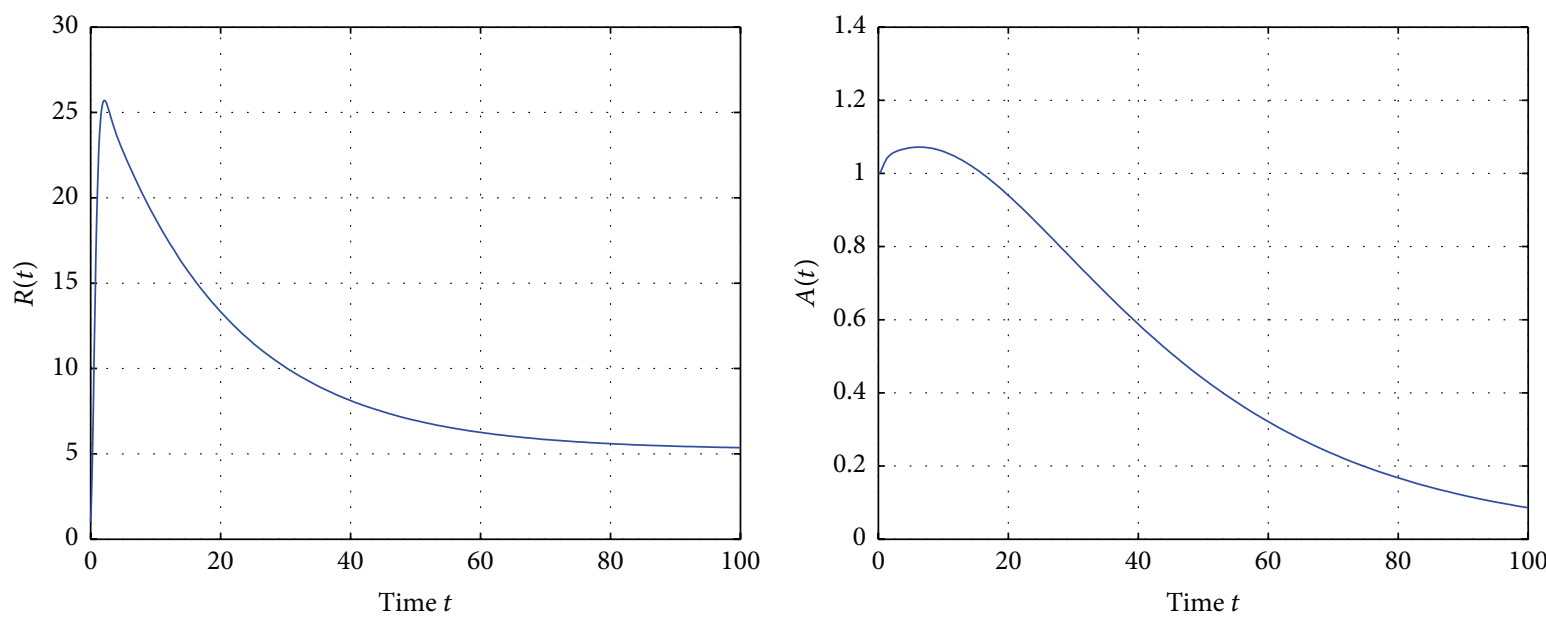

$-R(t)$

$-A(t)$

(c)
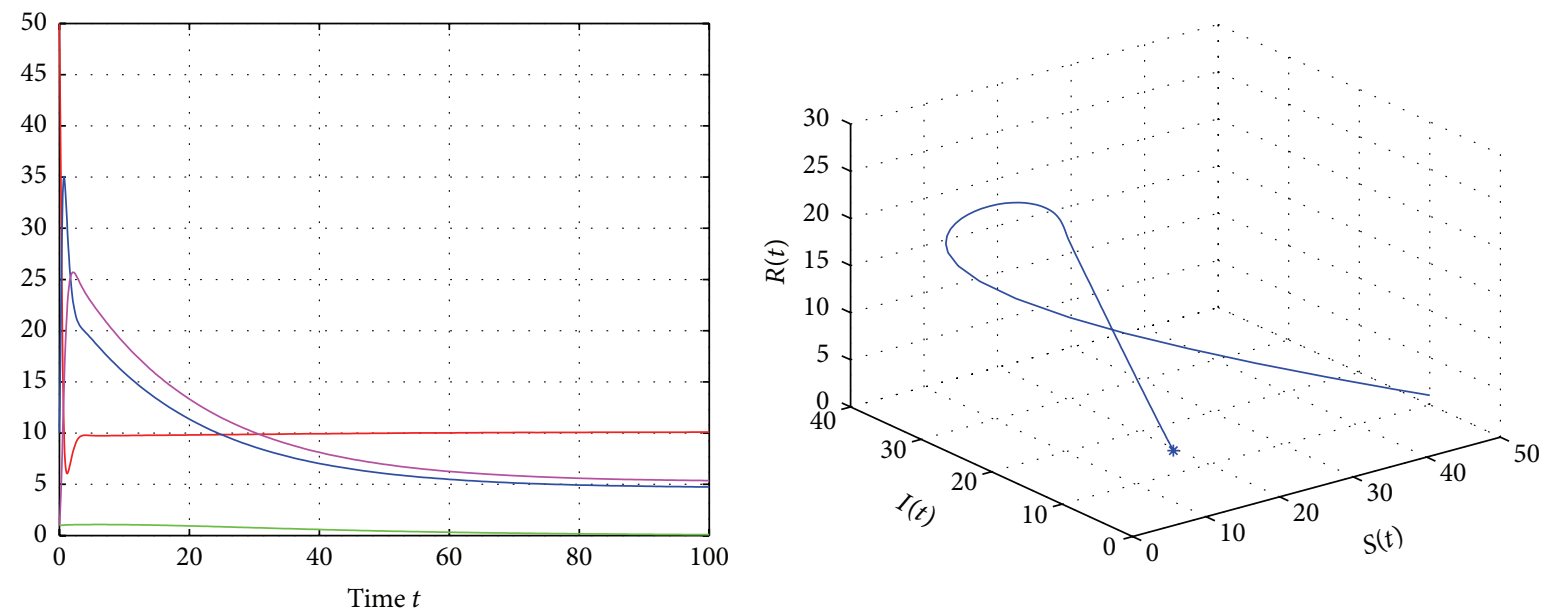

(e)

(f)

FIgURE 4: $1<R_{02}<R_{07}$. The positive equilibrium $P_{3}$ of system (1) is asymptotically stable. 


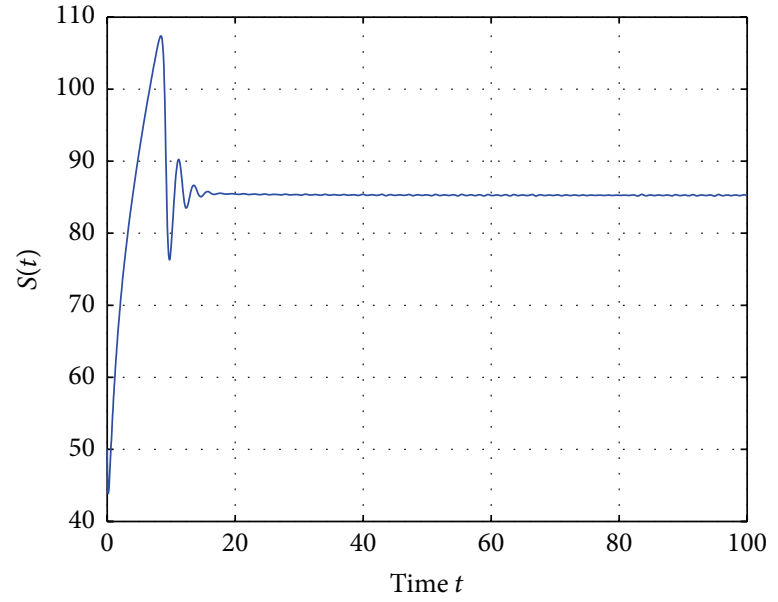

$-S(t)$

(a)

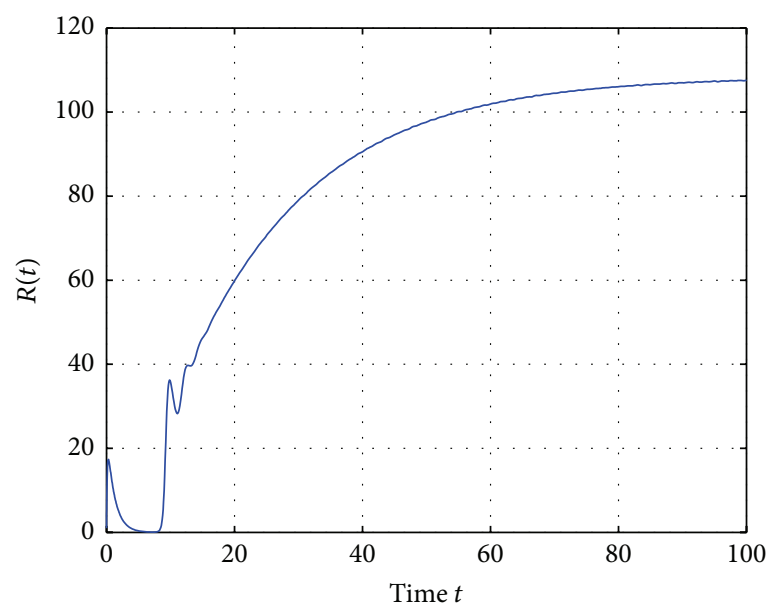

$-R(t)$

(c)

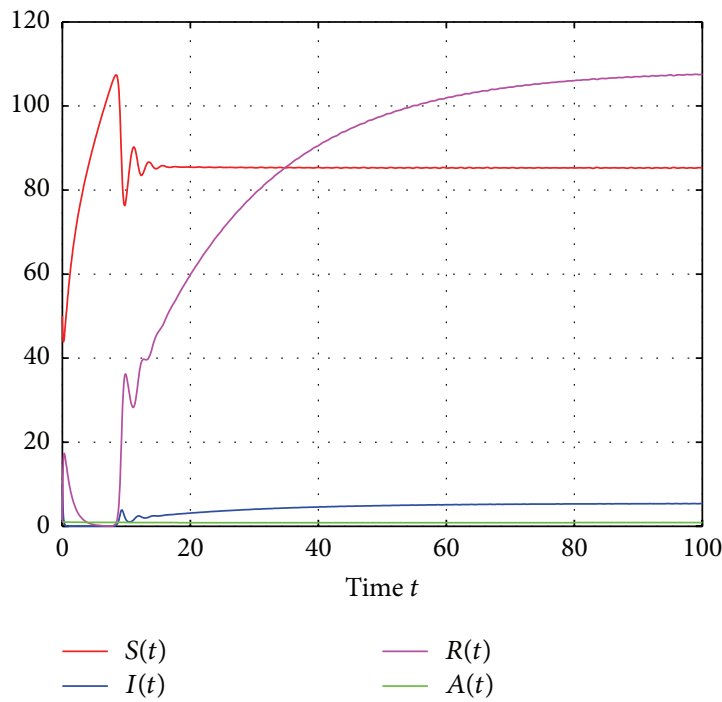

(e)

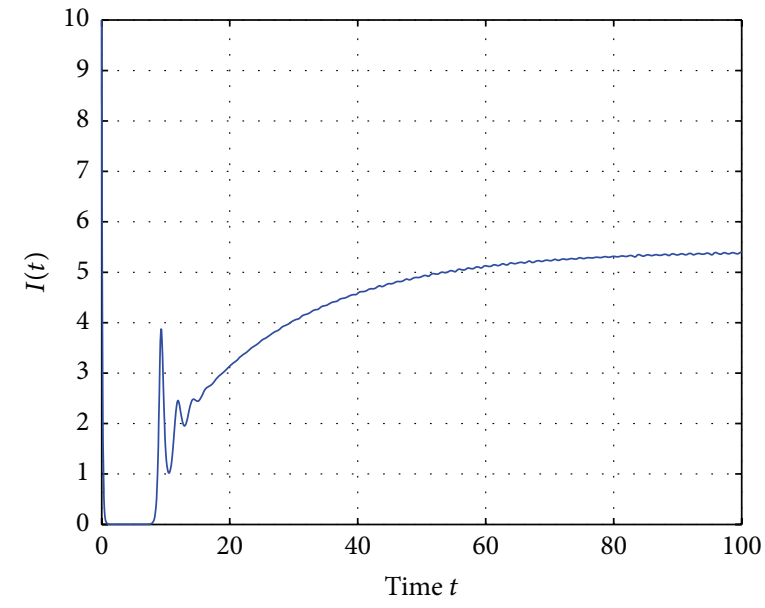

$-I(t)$

(b)

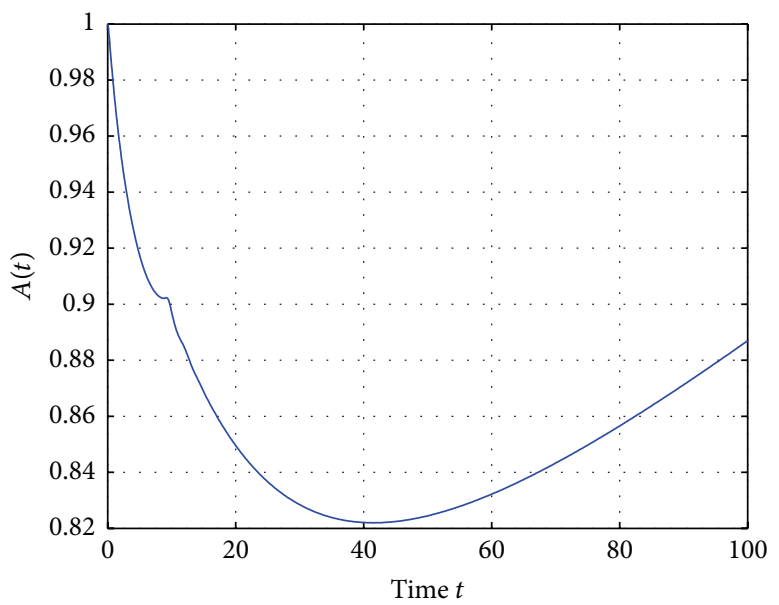

$-A(t)$

(d)

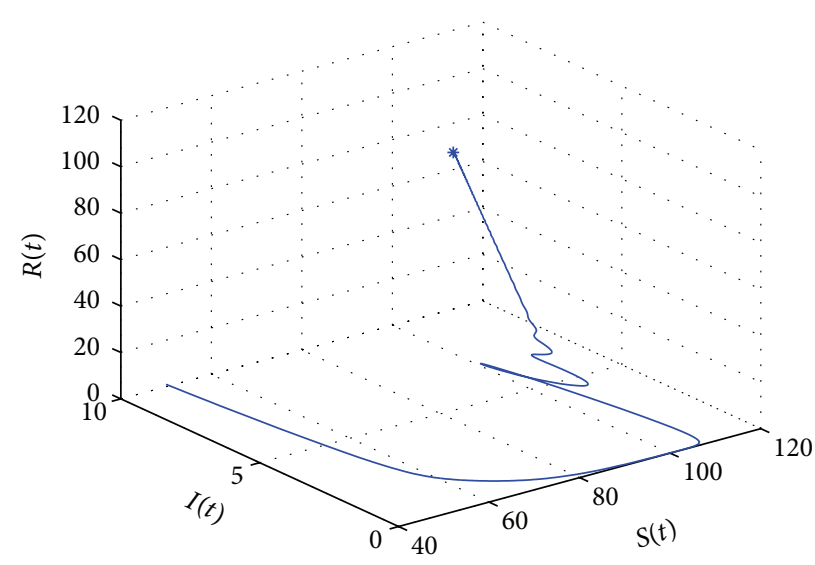

(f)

FIGURE 5: $1+\left(C \alpha_{I A}(\sigma+\mu)+\sigma \delta \mu\right) / \mu(\sigma+\mu)(\delta+\mu)<R_{03}<1+\left(\beta \mu /(\delta+\mu) \alpha_{S A}\right)\left(\left(C \alpha_{I A}(\sigma+\mu)+\sigma \delta \mu\right) / \mu(\sigma+\mu)(\delta+\mu)\right)$, and $R_{06}=$ $(\sigma+\mu)\left(\beta+\alpha_{I A}\right) / \alpha_{S A}(\sigma+\delta+\mu)>1$. The positive equilibrium $P_{4}$ of system (1) is asymptotically stable. 
Through the qualitative analysis of computer virus propagation model, mastering the virus prevention and control technology is very necessary. In the meantime, computer users have been advised to update their security settings. We can strengthen the knowledge of the computer virus spread (e.g., enhance the user's information security awareness) in a timely manner to install antivirus software or fix bugs, to minimize the impact on network computer virus.

\section{Conflict of Interests}

The author declares that there is no conflict of interests regarding the publication of this paper.

\section{Acknowledgments}

This work is partially supported by the National Natural Science Foundation of China (11301491) and the Youth Science Fund of Shanxi Province (2011021001-2).

\section{References}

[1] F. Cohen, "Computer viruses: theory and experiments," Computers \& Security, vol. 6, no. 1, pp. 22-35, 1987.

[2] J. O. Kephart and S. R. White, "Directed-graph epidemiological models of computer viruses," in Proceedings of the IEEE Computer Society Symposium on Research in Security and Privacy, pp. 343-358, May 1991.

[3] J. O. Kephart and S. R. White, "Measuring and modeling computer virus prevalence," in Proceedings of the IEEE Computer Society Symposium on Research in Security and Privacy, pp. 2-15, IEEE, Oakland, Calif, USA, May 1993.

[4] B. K. Mishra and N. Jha, "Fixed period of temporary immunity after run of anti-malicious software on computer nodes," Applied Mathematics and Computation, vol. 190, no. 2, pp. 12071212, 2007.

[5] B. K. Mishra and D. K. Saini, "SEIRS epidemic model with delay for transmission of malicious objects in computer network," Applied Mathematics and Computation, vol. 188, no. 2, pp. 14761482, 2007.

[6] J. R. C. Piqueira, B. F. Navarro, and H. A. M. Luiz, "Epidemiological models applied to viruses in computer networks," Journal of Computer Science, vol. 1, no. 1, pp. 31-34, 2005.

[7] J. R. Piqueira and V. O. Araujo, "A modified epidemiological model for computer viruses," Applied Mathematics and Computation, vol. 213, no. 2, pp. 355-360, 2009.

[8] J. R. C. Piqueira, A. A. de Vasconcelos, C. E. C. J. Gabriel, and V. O. Araujo, "Dynamic models for computer viruses," Computers and Security, vol. 27, no. 7-8, pp. 355-359, 2008.

[9] F. W. Wang, Y. K. Zhang, C. G. Wang, J. F. Ma, and S. Moon, "Stability analysis of a SEIQV epidemic model for rapid spreading worms," Computers and Security, vol. 29, no. 4, pp. 410-418, 2010.

[10] B. K. Mishra and N. Jha, "SEIQRS model for the transmission of malicious objects in computer network," Applied Mathematical Modelling, vol. 34, no. 3, pp. 710-715, 2010.

[11] B. K. Mishra and S. K. Pandey, "Fuzzy epidemic model for the transmission of worms in computer network," Nonlinear Analysis: Real World Applications, vol. 11, no. 5, pp. 4335-4341, 2010.
[12] B. K. Mishra and G. M. Ansari, "Differential epidemic model of virus and worms in computer network," International Journal of Network Security, vol. 14, no. 3, pp. 149-155, 2012.

[13] Z. Zhang and H. Yang, "Hopf bifurcation of an SIQR computer virus model with time delay," Discrete Dynamics in Nature and Society, vol. 2015, Article ID 101874, 8 pages, 2015. 


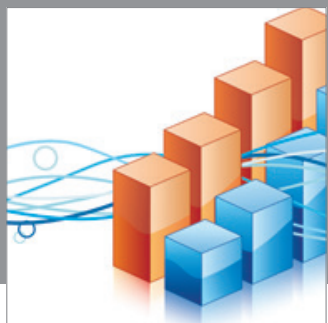

Advances in

Operations Research

mansans

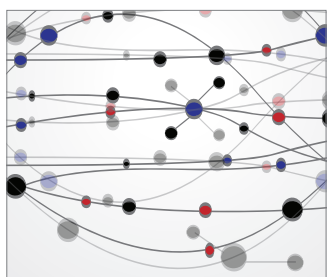

The Scientific World Journal
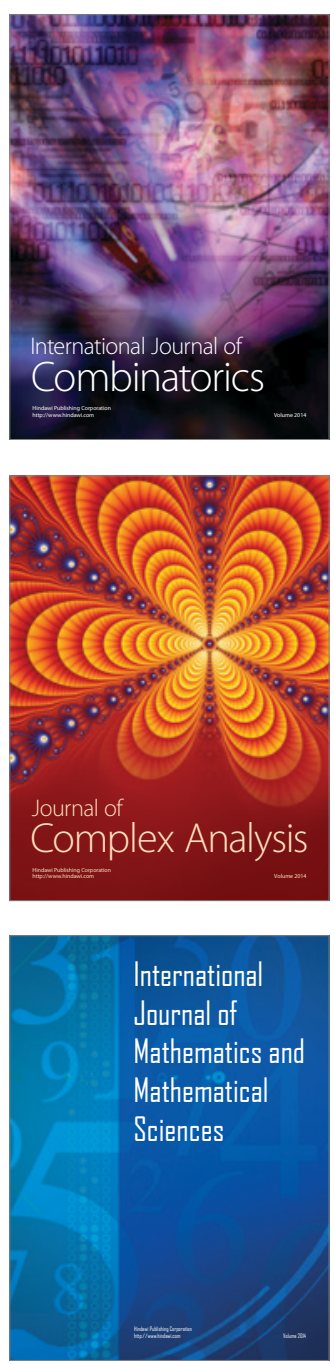
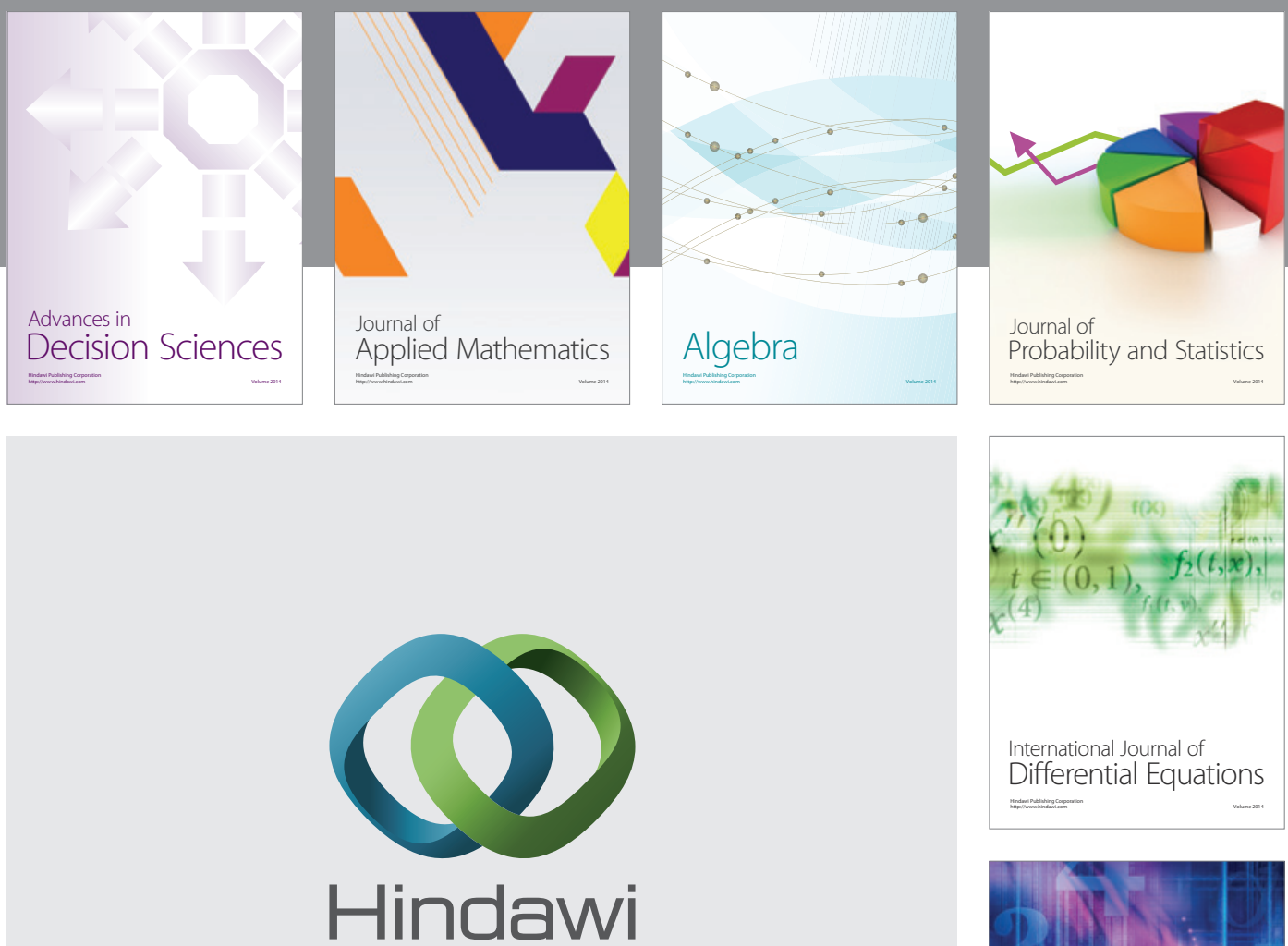

Submit your manuscripts at http://www.hindawi.com
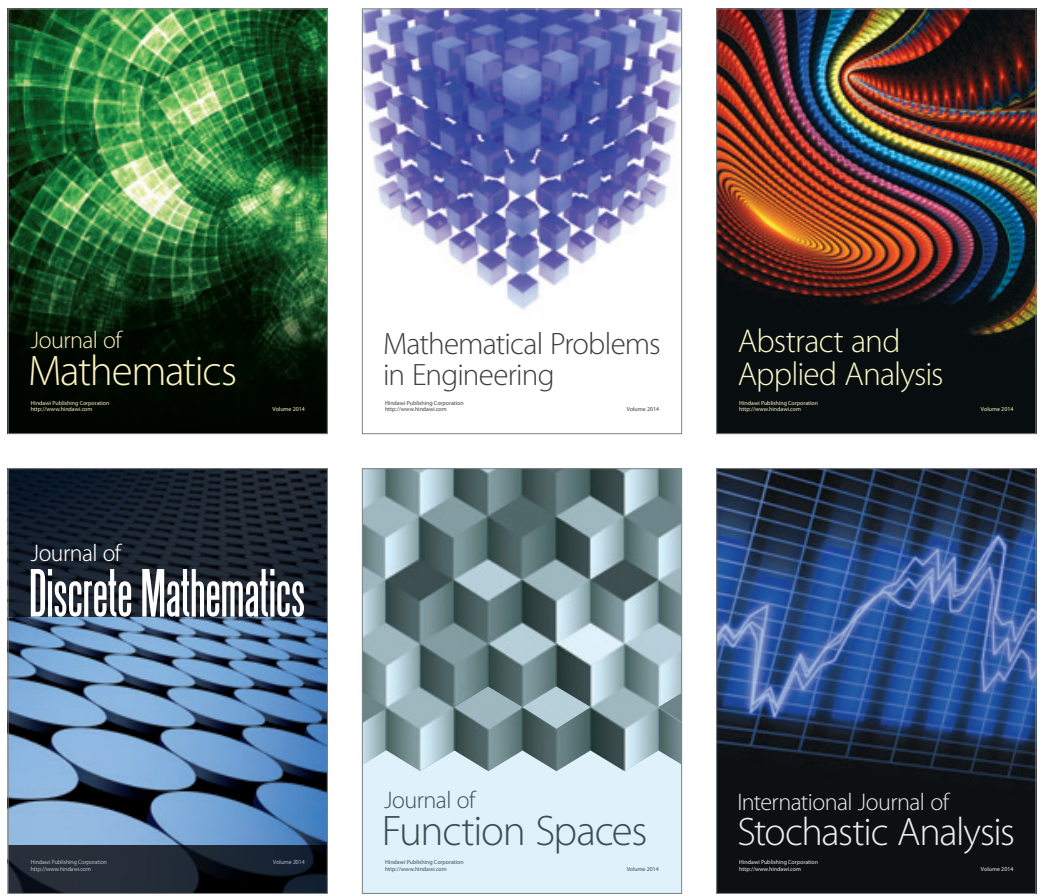

Journal of

Function Spaces

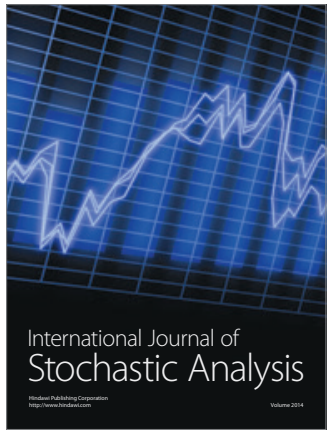

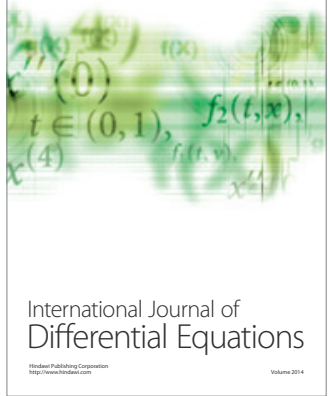
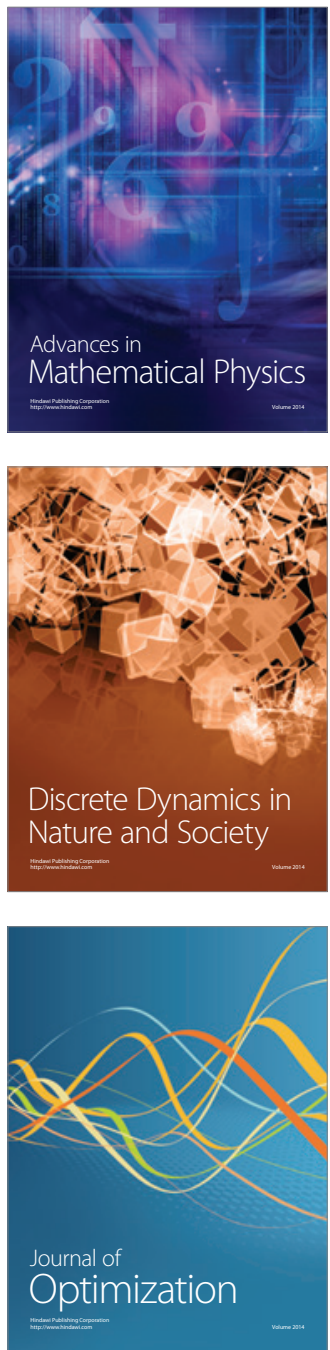